\title{
The effect of unpredicted visual feedback on activation in the secondary somatosensory cortex during movement execution
}

Toshiaki Wasaka* and Ryusuke Kakigi

\begin{abstract}
Background: A mechanism that monitors the congruence between sensory inputs and motor outputs is necessary to control voluntary movement. The representation of limb position is constantly updated on the basis of somatosensory and visual information and efference copy from motor areas. However, the cortical mechanism underlying detection of limb position using somatosensory and visual information has not been elucidated. This study investigated the influence of visual feedback on information processing in somatosensory areas during movement execution using magnetoencephalography. We used an experimental condition in which the visual information was incongruent despite the motor execution and somatosensory feedback being congruent. Subjects performed self-paced bimanual movements of both thumbs, either symmetric or asymmetric, under normal visual and mirrored conditions. The mirror condition provided a visual feedback by showing a reflection of the subject's right hand in place of the left hand. Therefore, in the Asymmetric task of the Mirror condition, subjects saw symmetric movements despite performing asymmetric movements.
\end{abstract}

Results: Activation in the primary somatosensory area (SI) revealed inhibition of neural activity and that in the secondary somatosensory area (SII) showed enhancement with voluntary movement. In addition, the SII contralateral to the side of stimulation was significantly enhanced in the Asymmetric task of the Mirror condition, which provided non-veridical visual feedback.

Conclusions: These results suggested that visual information influenced the neuronal activity concerning sensorimotor interaction in the SII during motor execution. The SII contributes to the detection of unpredicted visual feedback of movement execution.

Keywords: MEG, Sensorimotor integration, Bimanual movement, SII

\section{Background}

The information for movement is provided by visual and somatosensory feedback and the integration of crossmodal sensory processing and motor command is critical for motor control. Efficient monitoring of body movement depends on matching the predicted sensory consequences from internal motor commands with actual sensory feedback $[1,2]$. Normally, visual estimates of limb positions are congruent with somatosensory estimates under normal visual conditions, but a mismatch between watched and felt movements of the hand

\footnotetext{
* Correspondence: wasaka@nips.ac.jp

Department of Integrative Physiology, National Institute for Physiological Sciences, 38 Nishigonaka, Myodaiji, Okazaki, Aichi 4448585, Japan
}

disrupts motor control. This situation typically produces relatively strong cognitive conflict such as the feeling that movement is not controlled by oneself.

Recently, an increasing number of studies have indicated that cross-modal interaction occurred when neural activity from one sensory modality modulated activity in another [3-5]. Cross-modal links between visual and somatosensory information have shown the critical role of vision in determining limb position and localizing tactile sensations [6-9]. For example, viewing a body part improves tactile perception and facilitates the amplitude of long-latency components of event-related potentials [10-12]. In addition, there is evidence that vision of the body is crucial for localization of tactile stimuli $[13,14]$. These results indicate that the 
visual information changes the information processing in somatosensory areas.

Although less attention has been devoted to the effect of observation of movement on information processing in somatosensory areas, some studies have reported neural modulation in the primary somatosensory area (SI) and secondary somatosensory area (SII). Previous studies showed that viewing another person's gestures modulates the excitability of somatosensory areas [15-18]. These results indicate that the somatosensory areas are involved in the mirroring of actions. In addition, our recent study showed that somatosensory areas have a functional role to detect a mismatch between the intended and actual visual feedback of voluntary movement [19]. However, we could not fully clarify the neural mechanism of cross-modal interaction between visual and somatosensory modalities during movement execution. We must look more carefully into the effect of visual feedback on somatosensory information processing because the mismatch was very different between the expected and actual visual information in previous study. In the Mirror condition, which provided false visual information on movement, the actual visual feedback was a stationary hand and the intended feedback was an image of a moving thumb. To further address this issue, the present study investigated the neural modulation caused by the visual feedback that only differed in the phase of the moving hand.

The aim of the present study was to elucidate sensorimotor mechanisms in detecting incongruence if visual feedback did not match with the predicted one based on somatosensory feedback and motor command. We experimentally manipulated visual feedback using the mirror box technique [20-22]. We created non-intended visual feedback by showing a reflection of the subject's right hand in place of the left hand during movement execution.

\section{Methods \\ Subjects}

Ten healthy volunteers participated in the study (10 males; mean age $35.2 \pm 5.3$ years, range $28-46$ years). None had a history of neurological disorders or took medication before the experiment. All subjects were right-handed as assessed by the Edinburgh Handedness Inventory (average score, 91.1) [23]. Written informed consent was obtained from each participant prior to the study, which was approved by the Ethics Committee at the National Institute for Physiological Sciences, Okazaki, Japan.

\section{Stimulation}

The left median nerve was stimulated on the palm side at the wrist with a saddle-type electrode. The electrode was fixed to the wrist using a belt to prevent it moving during movement execution. The cathode was placed
$3 \mathrm{~cm}$ proximal to the anode. The electrical stimulus was a constant current square-wave pulse $0.2 \mathrm{~ms}$ in duration and the stimulus intensity was 1.1 times the motor threshold of the abductor pollicis brevis muscle, which yielded no pain or unpleasant sensation. The interstimulus interval of electrical stimuli was $3000 \mathrm{~ms}$.

\section{Experimental conditions}

In this study, we measured brain activities in somatosensory areas while subjects performed two bimanual movement tasks in two conditions (Figure 1). In the Mirror condition, subjects inserted their hands into a mirror box with the forearm supine. The position of the right hand was adjusted so that the mirror image precisely overlapped the view of the masked left hand. A mirror image of the right hand was therefore presented instead of the left hand. In the NoMirror condition, the mirror box was removed. For the magnetoencephalography (MEG) recordings, subjects were instructed to gaze at a small round mark $(0.8 \mathrm{~cm}$ in diameter $)$ approximately $0.5 \mathrm{~m}$ away between the two hands.

In each condition, three tasks were performed. Subjects were instructed to make simple, self-paced repetitive hand movements that involved extending and flexing both thumbs either symmetrically (Symmetric task) or asymmetrically (Asymmetric task). In both tasks, we asked the subjects to perform the sequence of movement (thumb flexion-extension) at a frequency of about $1 \mathrm{~Hz}$. We asked that they did not synchronize their movements with the electrical stimulation. We monitored the thumb movement with a video camera from outside of a shielded room. If the pace of movement was slower or faster than $1 \mathrm{~Hz}$, we gave instructions to the subject. The other task was that both hands were kept stationary (Stationary task). In all tasks, subjects were instructed to pay no attention to the electrical stimulation. In the Mirror condition of the Asymmetric task, since the actual visual information on left hand movement was masked by the mirror, subjects saw symmetric movements despite performing asymmetric movements. In contrast, for the Symmetric task, although subjects saw the mirrored movement, the phase of bimanual movement was the same in masked and mirrored hands. One session of the three tasks comprised 50 stimuli and the recording time of one session was about $3 \mathrm{~min}$. Two sessions were performed, and 100 artifact-free responses were averaged for each condition. The order of tasks in each condition was randomized among the subjects.

\section{Data acquisition and analysis}

The MEG signals were recorded with a helmet-shaped 306-channel detector array (Vectorview, Elekta Neuromag Yo, Helsinki, Finland), which comprised 102 identical triple sensor elements. Each sensor element consisted of two 


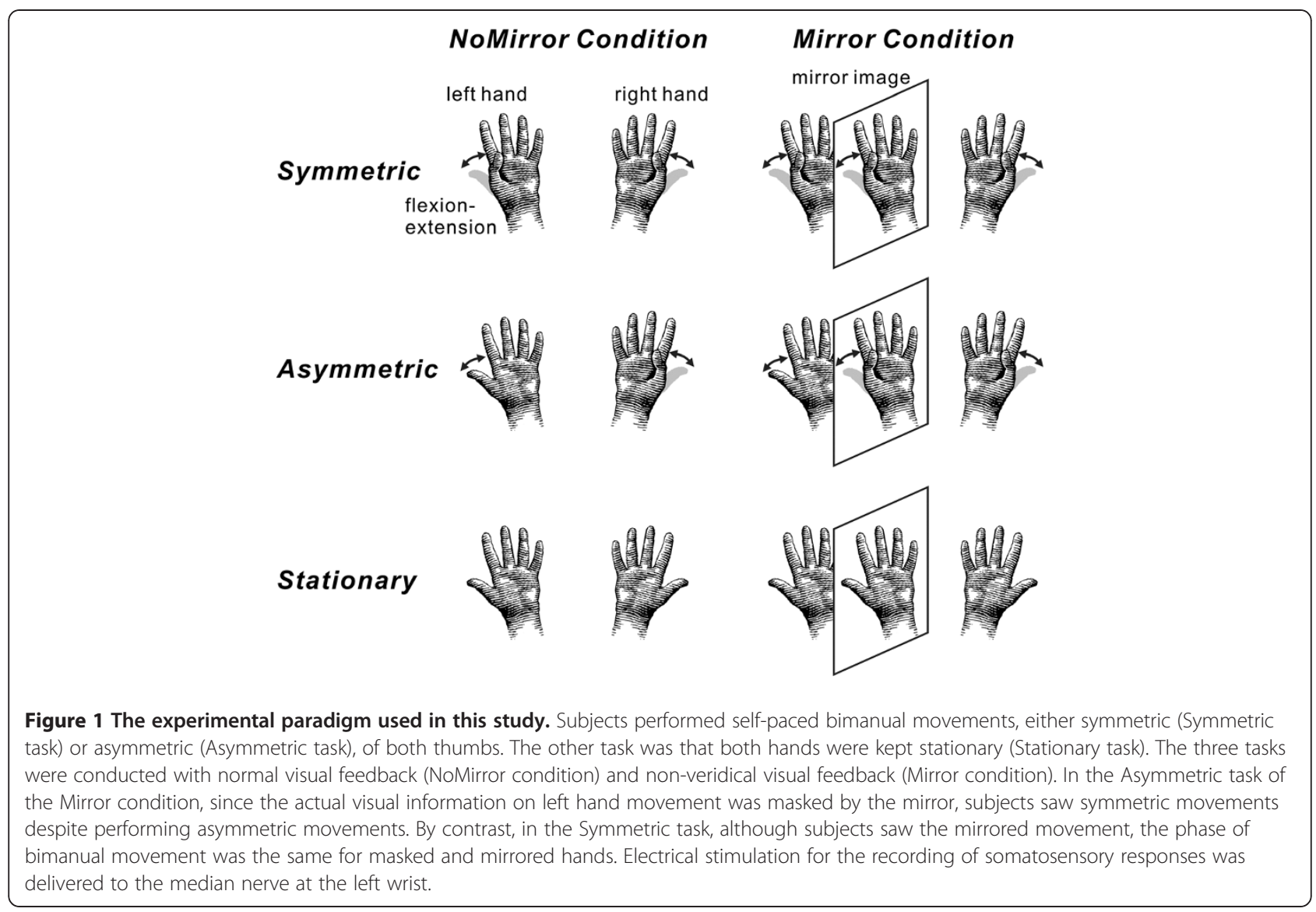

orthogonal planar gradiometers and one magnetometer coupled to a multi-superconducting quantum interference device and thus provided three independent measurements of the magnetic fields. The MEG signals were recorded with a bandpass filter of $0.03-300 \mathrm{~Hz}$ and digitized at $1024 \mathrm{~Hz}$. Epochs in which the signal variation was larger than $3000 \mathrm{fT} / \mathrm{cm}$ were excluded from the averaging. The period of analysis was from $100 \mathrm{~ms}$ before to $250 \mathrm{~ms}$ after the onset of the electrical stimulus. The data for $100 \mathrm{~ms}$ before the stimulus were used to calculate the baseline.

Prior to the MEG recording, four head position indicator (HPI) coils were placed at specific sites on the scalp. To determine the exact location of the head with respect to the MEG sensors, an electric current was fed to the HPI coils, and the resulting magnetic fields were measured with the MEG sensors. These procedures allowed for alignment of the individual head coordinate system with the MEG coordinate system. The locations of HPI coils with respect to the three anatomical landmarks (nasion and bilateral preauriculas) were also measured using a three-dimensional digitizer to align the coordinate systems of MEG with the T1weighted magnetic resonance images (MRI) obtained with a 3 tesla MRI system (Allegra; Siemens, Erlangen, Germany). The $\mathrm{x}$-axis was fixed with the preauricular points, the positive direction being to the right. The positive $y$-axis passed through the nasion and the $\mathrm{z}$-axis thus pointed upward.

We first calculated vector sums from the longitudinal and latitudinal derivatives of responses recorded on the planar-type gradiometers at each of the 102 sensors' locations (Figure 2). This was achieved by squaring MEG signals for each of two planar-type gradiometers at a sensor's location, summing the squared signals together, and then calculating the root of the sum, here we call this the "root sum square" (RSS) [24]. The calculation was carried out for all 102 sensors' locations. Next, we used the RSS waveforms and a topographic map of RSS amplitude to look for a peak channel showing the greatest amplitude for each prominent response, because the waveforms had several responses with a different spatial distribution of amplitude. Then, the peak amplitude and latency of prominent responses in the RSS waveform were measured at the peak channel.

To identify the equivalent current dipoles (ECDs) in MEG components, sources of measured responses to the electrical stimuli were modeled with the timevarying current dipoles method [25]. Single ECDs of MEG components were estimated. In the period when clear dipolar magnetic field patterns were found, the 


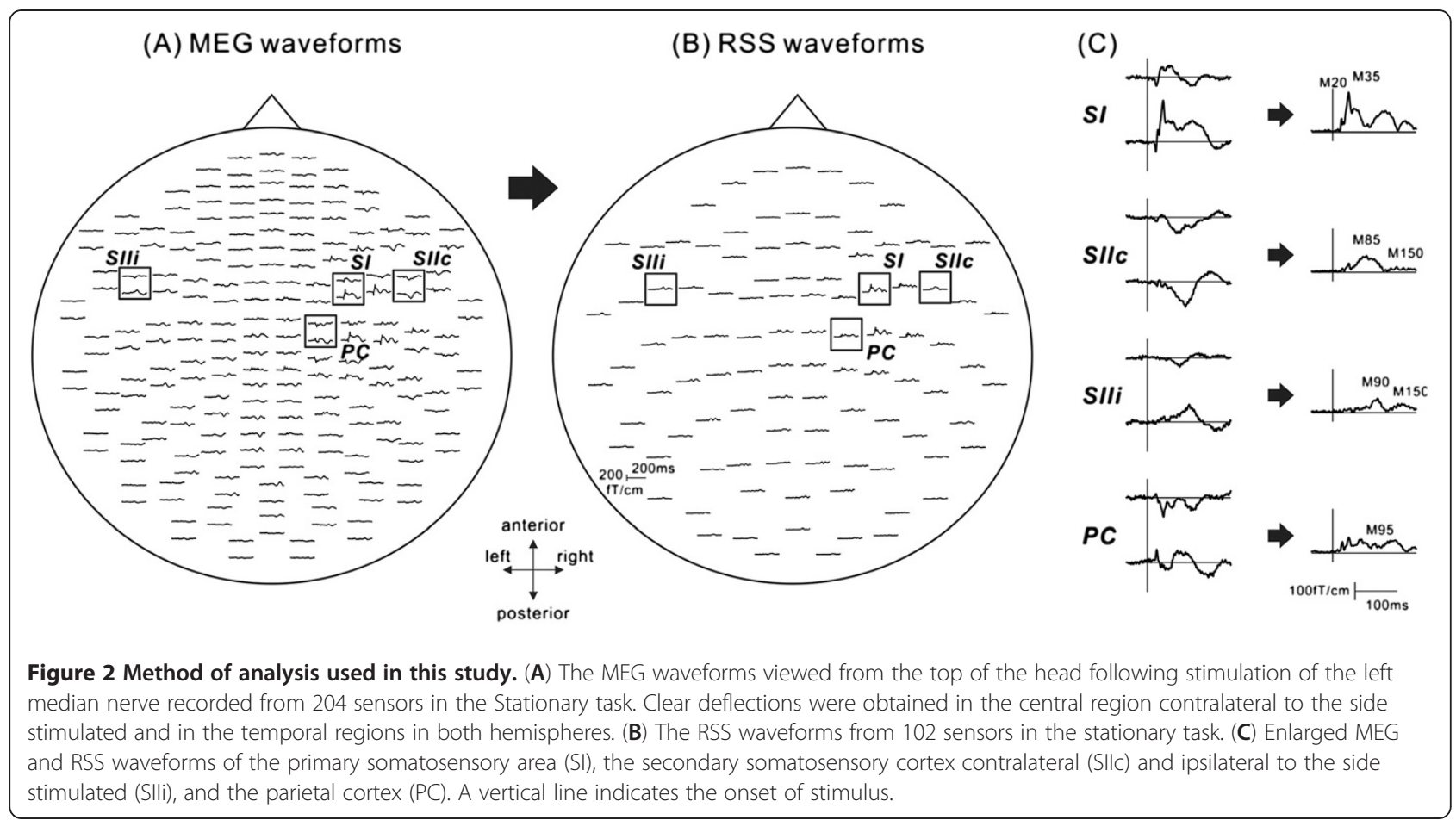

ECDs that best explained the most dominant signals were determined by a least-squares search, based on 14 to 18 channels around the channel that had been used to measure the peak amplitude of RSS waveforms. The goodness-of-fit (GOF) value of an ECD was calculated to indicate in percentage terms how much the dipole accounted for the measured field variance. Only ECDs which accounted for more than $80 \%$ of the GOF in a channel subset were accepted.

For the peak amplitude of the RSS components, to examine whether visual feedback affects the activation of somatosensory areas during bimanual movement, a twoway repeated measures ANOVA was performed with visual condition (Mirror and NoMirror conditions) and movement task (Symmetric, Asymmetric and Stationary tasks) as the factors. To analyze the assumption of sphericity prior to the repeated measures ANOVA, we used Mauchly's test of sphericity. If a significant test result was obtained and the assumption of sphericity was violated, the Greenhouse-Geisser adjustment was used to correct for the sphericity by altering the degrees of freedom using a correction coefficient epsilon. A posthoc analysis was conducted using paired t-test. Statistical significance was set at a P value of less than 0.05 .

\section{Results}

Figure 2 shows the waveforms of somatosensory evoked fields (SEFs) and RSS in the stationary condition following stimulation of the left median nerve. Five prominent components were observed in RSS waveforms. The earliest deflection of the RSS waveforms was identified in the central region contralateral to the side stimulated at around $20 \mathrm{~ms}$ after the stimulation (M20) and the subsequent deflection peaking at around $35 \mathrm{~ms}$ (M35). Long-latency components were identified in temporal regions of both hemispheres at around $90 \mathrm{~ms}$ (M85 and M90). The ECD for the early responses was located in the posterior bank of the central sulcus corresponding to the SI. The bilateral long-latency responses were estimated to be generated in the upper bank of the sylvian fissure, corresponding to the SII (Figure 3). In addition, around the central region contralateral to the side of stimulation, the sensor was located more posterior than the SI, and a small peak was observed at about $95 \mathrm{~ms}$ (M95). The source of the M95 was estimated to be around the parietal cortex (PC) in the posterior wall of the postcentral sulcus.

Grand-averaged RSS waveforms for each task of the two conditions are shown in Figure 3. To investigate the effect of visual feedback on neural activation in the somatosensory areas during movement execution, we compared the peak amplitude of RSS components using a two-way repeated ANOVA concerning task (Symmetric, Asymmetric and Stationary) and condition (Mirror and NoMirror). Figure 4 shows the peak amplitude of RSS components. A significant main effect of task on the M20 and M35 in the SI was found (M20 $\mathrm{F}_{1,9}=7.875, \mathrm{P}<0.05 \mathrm{M} 35 \mathrm{~F}_{1,9}=16.093, \mathrm{P}<0.01$ ), but no significant main effect of condition or interaction. The amplitude of SI components exhibited a 

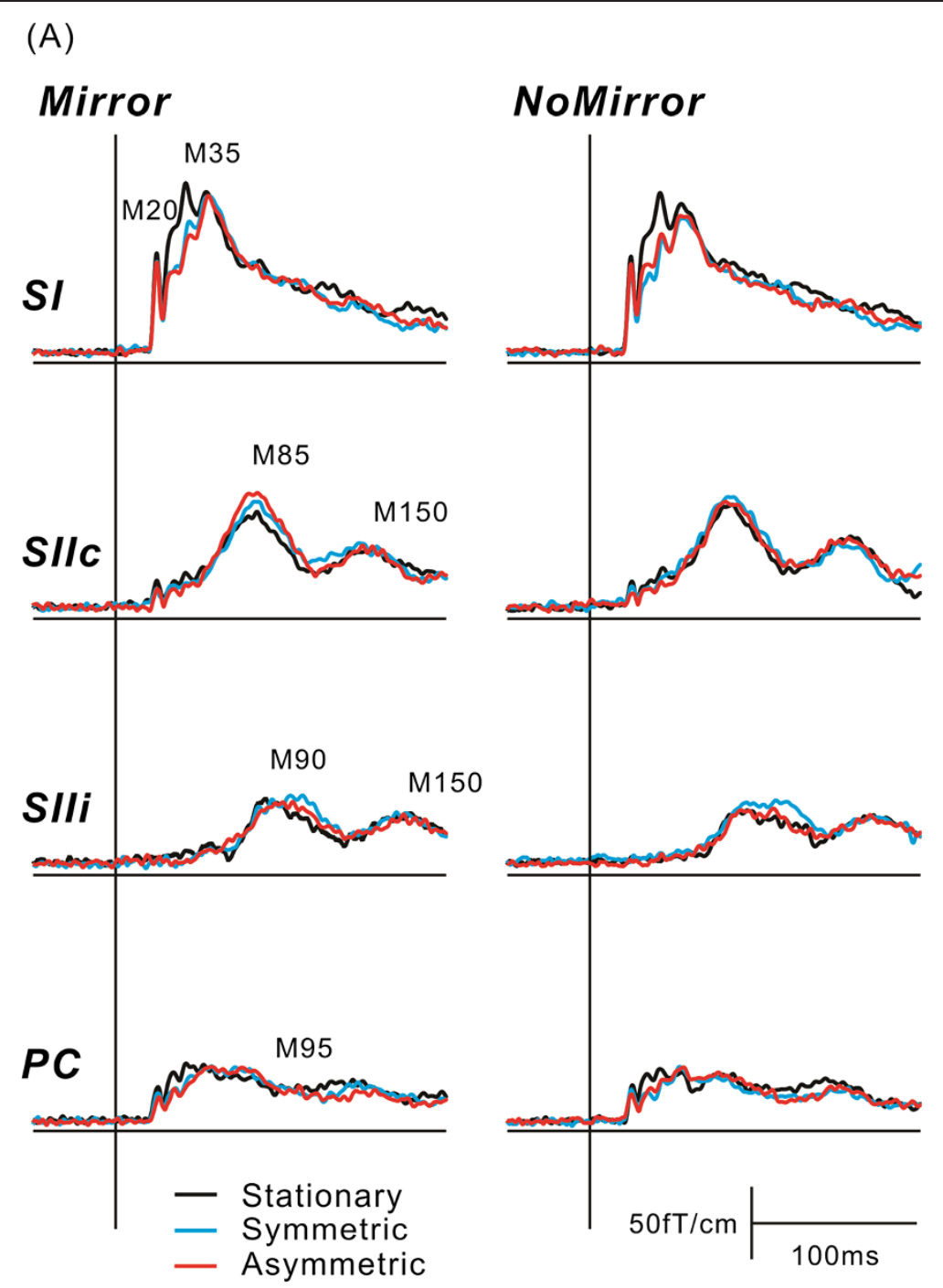

(B)
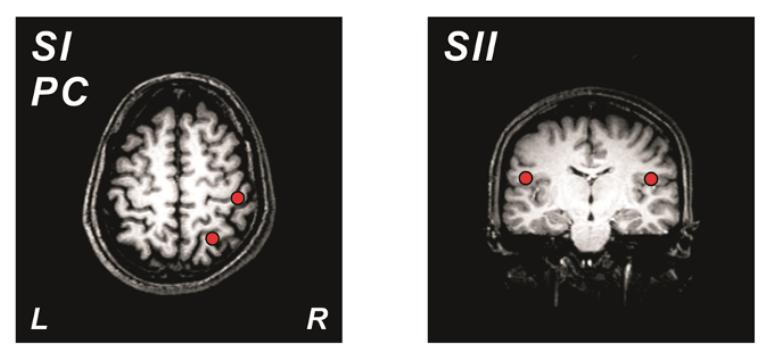

Figure 3 The grand averaged RSS waveforms and dipole locations. (A) The grand averaged RSS waveforms in each task of the two conditions in the SI, Sllc, Slli, and PC. A vertical line indicates the onset of stimulus. Prominent responses were observed at around $20 \mathrm{~ms}$ (M20) and $35 \mathrm{~ms}$ (M35) in the SI, around 85 ms (M85) and $150 \mathrm{~ms} \mathrm{(M150} \mathrm{Sllc)} \mathrm{in} \mathrm{the} \mathrm{Sll} \mathrm{contralateral} \mathrm{to} \mathrm{the} \mathrm{side} \mathrm{of} \mathrm{stimulation,} \mathrm{around} 90$ ms (M90) and 150 ms (M150 Slli) in the SII ipsilateral to the side stimulated and around 95 ms (M95) in the PC. A reduction in SI components and an enhancement of SII components were identified with movement execution. In addition, the M85 showed significant enhancement in the Asymmetric task of the Mirror condition. (B) The location of equivalent current dipoles (ECDs) in each component superimposed on the T1weighted magnetic resonance images. The ECD for short-latency responses was located in the posterior bank of the central sulcus (SI) in the hemisphere contralateral to the side stimulated, and the long-latency responses (M85 and M90) were identified in the bilateral temporal regions around the upper bank of the sylvian fissure, corresponding to the SII. The source of the parietal activity (M95) was located medial and posterior to the SI hand area in the hemisphere contralateral to the side stimulated. 

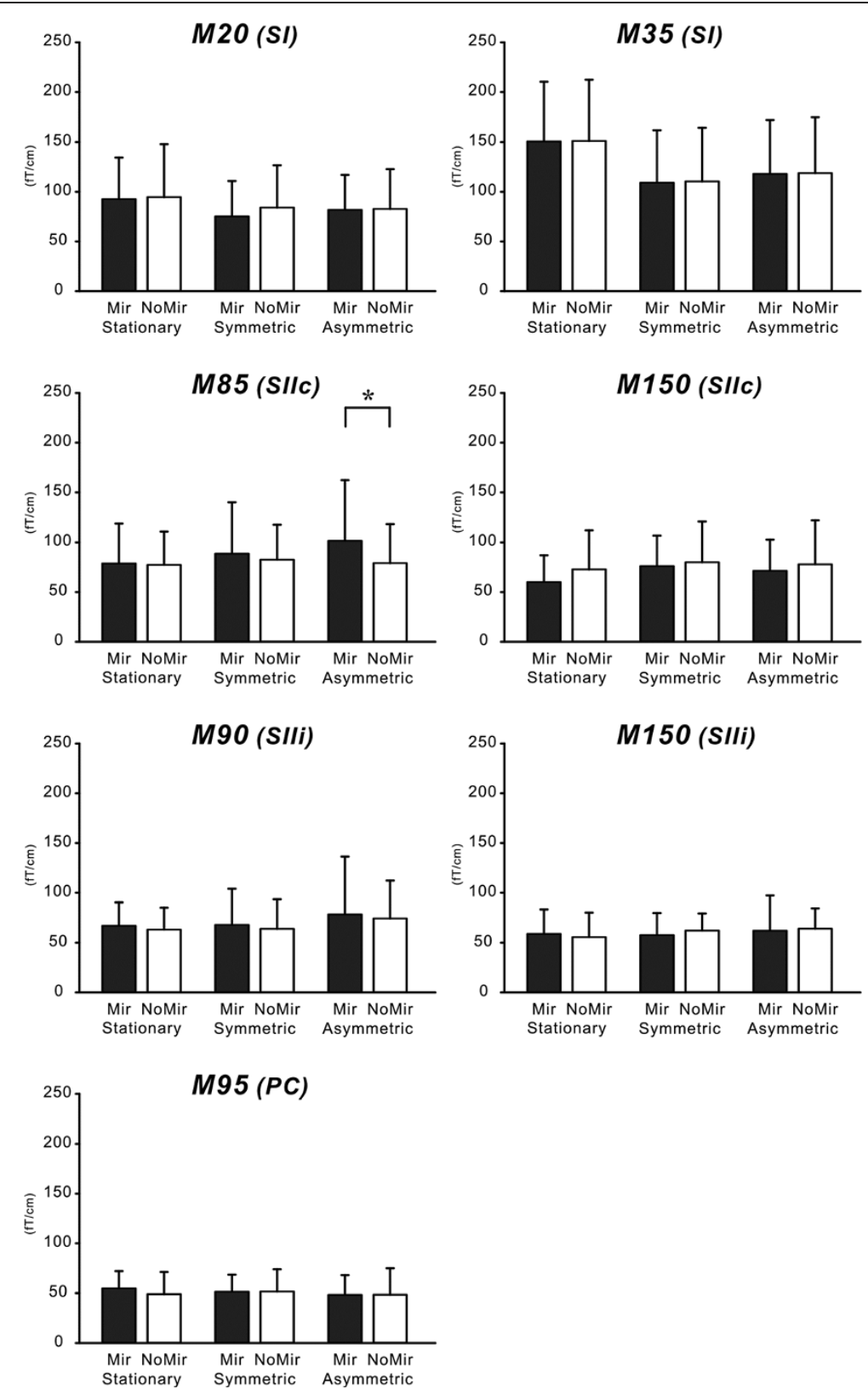

Figure 4 The modulation of RSS components with voluntary movement in the Mirror and NoMirror conditions. The $y$-axis indicates the peak amplitude of RSS component and the error bar means the standard deviation. A two-way repeated measures ANOVA was performed with visual condition (Mirror and NoMirror conditions) and movement task (Symmetric, Asymmetric and Stationary tasks) as the factors. A significant main effect of task was found in the M20 and M35 of the SI. In addition, a significant interaction was found in the M85 of SIlc and M95 of PC. The amplitude of the M85 in the Asymmetric task was significantly larger in the Mirror condition than NoMirror condition. In contrast, no difference was observed in the Symmetric task. ${ }^{*} P<0.05$, between two conditions.

reduction during movement execution. In the SII contralateral to the side of stimulation (SIIc) and PC components, although no significant main effect was observed, a significant interaction was found in the M85 $\left(\mathrm{F}_{2,18}=6.425, \mathrm{P}<0.05\right)$ and M95 $\left(\mathrm{F}_{2,18}=3.731, \mathrm{P}<0.05\right)$. In contrast, no significant change was observed in the M90 and M150 in the SII ipsilateral to the side of stimulation (SIIi) and the M150 in the SIIc. We compared the SIIc component in each task between the Mirror and NoMirror conditions. In the Asymmetric task, the amplitude of the SIIc was significantly higher in the Mirror than NoMirror condition $(\mathrm{P}<0.05)$, while no significant change was observed in the Stationary and Symmetric tasks between the two visual conditions. 
The non-veridical visual feedback in the Asymmetric task of the Mirror condition affected the activation of the SIIc.

\section{Discussion}

We investigated whether activation in somatosensory areas was affected by the discordance of visual information between intended and executed actions. Subjects received visual feedback about thumb movement that was unintended in the Asymmetric task of the Mirror condition or expected in the Symmetric task of the Mirror condition and NoMirror condition. In the conflict caused by the unintended visual feedback, activation in the SIIc was significantly higher in the Mirror condition than NoMirror condition. The results of this study are in line with our previous findings showing cross-modal interaction between somatosensory and visual modalities in the SII when looking at unintended visual information on a moving hand provided during movement execution [19].

Viewing the body influences the speed of tactile reaction time [26] and improves tactile acuity [27]. Longo et al. [28] reported that the short-latency component of somatosensory evoked potentials (SEPs) generated in the SI was higher on viewing the body than viewing an object. Furthermore, in addition to viewing the body, the observation of moving body parts also modulates the SI activation [15-18], whereas our results showed no changes of SI activity on viewing the unintended visual feedback of body movement. It is well-known that the neural activity in the SI is strongly inhibited during voluntary movement [29-34]. One explanation for the absence of modulation of SI activity with the unexpected visual information in our study may be that the inhibitory effect from motor-related areas canceled out the effect from visual information.

We found that the SIIc response in the Asymmetric task was significantly higher in the Mirror condition than NoMirror condition, while no change was observed in the Symmetric task. The Asymmetric task in both conditions was similar with regard to motor command and somatosensory feedback, and the only difference was the visual feedback. There was a possibility that a very subtle twitch following the stimulation caused the difference in visual feedback between the Mirror and NoMirror conditions. However, since the stimulus intensity was just above the motor threshold, the extensionflexion of the thumb was much larger than the small twitch during movement tasks. It seems reasonable to eliminate the notion that the difference in visual feedback caused by a minor twitch induces the enhancement in the SIIc.

The neural mechanism responsible for modulation of SIIc activities through non-veridical visual feedback is unclear. One possibility is that the incongruent visual information enhances the SIIc activation via an attentional effect. The SII is involved in cross-modal attentional links between the somatosensory and visual modalities [3], and the sight of body parts influences somatosensory event-related potentials with tactile spatial attention [14]. A probable explanation for the modulated neural activations in the SIIc observed in the Mirror condition might be that the visual feedback of unintended phase movement caused by replacement of the subjects' left hand with a mirror image of the right hand implicitly led to increased attentional demands for the somatosensory information. Another possibility is that the neural mechanism providing the visual information on the body part would be influenced by predictions of visual feedback based on the motor commands. It has been suggested that a copy of the motor signal, known as an efference copy, is created so that sensory signals generated from external stimuli can be distinguished from reafferent signals from body movement $[2,35,36]$. Corollary discharges are produced only if the motor commands interact with unpredicted sensory inputs and inhibits the neural response to the self-generated sensory signals [37]. More activity in somatosensory areas was found when the unpredicted stimulus was externally delivered [38-40]. There is considerable validity to notion that the prediction of visual feedback modulates the somatosensory areas. In the Asymmetric task in the Mirror condition, subjects faced the surprise of seeing that their hand was not responding as intended, and activation in the SIIc might be modulated by the effect of corollary discharge.

We assume that this modulation in the SIIc is involved in computing the sensory errors by comparing the actual hand's location to the estimated location for controlling movement. There is another cortical area that is important to a forward model [2]. The cerebellum builds internal models that predict the sensory feedback of motor commands and correct motor commands through internal feedback. The cerebellum also has been proposed to combine information from motor efferent and sensory afferent signals [41]. However, we could not record any cerebellar responses because our whole-head MEG system did not fully cover the cerebellum.

There is evidence that humans are normally not conscious of sensory feedback from movement [42], and are aware that their arms and legs belong to them through somatosensory and visual inputs. This feeling of selfattribution is impaired when the predicted sensory information estimated from motor intention does not match the actual sensory information. In our study, the Asymmetric task of the Mirror condition corresponded to this situation. Some subjects reported feeling that movement in the Asymmetric task of the Mirror condition was not controlled by themselves or the body did not belong to 
them. Studies in patients and recent neuroimaging results in healthy subjects suggest a prominent role for the posterior parietal cortex [43] and insula [44-47] in the self-awareness of limb actions, the sense of agency. Inui et al. [48] reported simultaneous activation in the contralateral SII and insula peaking at 90 to $160 \mathrm{~ms}$ after electrical stimulation. We assumed that the late component peaking at $150 \mathrm{~ms}$ (M150) in the SIIc may involve the SII activity of the neighboring insula. However, we could not find significant differences in the insula and PC. Although subjects reported a disturbance of agency, we assumed that it was not enough to induce the difference in these areas. Further study will be needed to clarify the functional role of these areas in sensorimotor integration.

\section{Conclusions}

This study revealed the neural mechanism of somatosensory activation during visual conflict caused by incongruence between the predicted and actual visual feedback in motor control. Our results demonstrated that the SII had cross-modal functions in the somatosensory and visual modalities during motor execution. However, we did not elucidate visuo-tactile interaction in other somatosensory areas such as the posterior parietal cortex and insula. Further research may be useful to elucidate the functional role of somatosensory areas for motor control using somatosensory and visual information.

\section{Authors' contributions}

TW contributed to planning the study, data collection, analysis, and drafting the paper. RK contributed to planning the study and drafting the paper. Both authors read and approved the final manuscript.

\section{Acknowledgements}

We thank Mr. Y. Takeshima for help in devising, constructing, and maintaining the equipment used in this study. This study is the result of "Development of biomarker candidates for social behavior" carried out under the Strategic Research Program for Brain Sciences by the Ministry of Education, Culture, Sports, Science and Technology of Japan. This study was supported by a Grant-in-Aid for Young Scientists (B) to T.W. (23700689).

Received: 14 June 2012 Accepted: 25 October 2012

Published: 5 November 2012

\section{References}

1. Shadmehr R, Krakauer JW: A computational neuroanatomy for motor control. Exp Brain Res 2008, 185:359-381.

2. Wolpert DM, Miall RC, Kawato M: Internal models in the cerebellum. Trends Cogn Sci 1998, 2:338-347.

3. Kida T, Inui K, Wasaka T, Akatsuka K, Tanaka E, Kakigi R: Time-varying cortical activations related to visual-tactile cross-modal links in spatial selective attention. J Neurophysiol 2007, 97:3585-3596.

4. Macaluso E, Frith CD, Driver J: Modulation of human visual cortex by crossmodal spatial attention. Science 2000, 289:1206-1208.

5. Schurmann M, Kolev V, Menzel K, Yordanova J: Spatial coincidence modulates interaction between visual and somatosensory evoked potentials. Neuroreport 2002, 13:779-783.

6. vanBeers RJ, Sittig AC, vanderGon JJD: How humans combine simultaneous proprioceptive and visual position information. Exp Brain Res 1996, 111:253-261.
7. Botvinick M, Cohen J: Rubber hands 'feel' touch that eyes see. Nature 1998, 391:756

8. Graziano MSA: Where is my arm? The relative role of vision and proprioception in the neuronal representation of limb position. $P$ Nat/ Acad Sci USA 1999, 96:10418-10421.

9. MonWilliams M, Wann JP, Jenkinson M, Rushton K: Synaesthesia in the normal limb. P Roy Soc B-Biol Sci 1997, 264:1007-1010.

10. Cardini F, Longo MR, Haggard P: Vision of the body modulates somatosensory intracortical inhibition. Cereb Cortex 2011, 21:2014-2022.

11. Kennett S, Spence C, Driver J: Visuo-tactile links in covert exogenous spatial attention remap across changes in unseen hand posture. Percept Psychophys 2002, 64:1083-1094.

12. Taylor-Clarke M, Kennett $S$, Haggard P: Vision modulates somatosensory cortical processing. Curr Biol 2002, 12:233-236.

13. Eimer $M$, Forster $B$, Fieger $A$, Harbich S: Effects of hand posture on preparatory control processes and sensory modulations in tactile-spatial attention. Clin Neurophysiol 2004, 115:596-608.

14. Sambo CF, Gillmeister H, Forster B: Viewing the body modulates neura mechanisms underlying sustained spatial attention in touch. Eur J Neurosci 2009, 30:143-150.

15. Avikainen S, Forss N, Hari R: Modulated activation of the human SI and SII cortices during observation of hand actions. Neurolmage 2002, 15:640-646.

16. Mottonen R, Jarvelainen J, Sams M, Hari R: Viewing speech modulates activity in the left SI mouth cortex. Neurolmage 2005, 24:731-737.

17. Pihko $E$, Nangini $C$, Jousmaki $V$, Hari R: Observing touch activates human primary somatosensory cortex. Eur J Neurosci 2010, 31:1836-1843.

18. Rossi S, Tecchio F, Pasqualetti P, Ulivelli M, Pizzella V, Romani GL, Passero S, Battistini N, Rossini PM: Somatosensory processing during movement observation in humans. Clin Neurophysiol 2002, 113:16-24.

19. Wasaka T, Kakigi R: Conflict caused by visual feedback modulates activation in somatosensory areas during movement execution. Neurolmage 2012, 59:1501-1507.

20. Altschuler EL, Wisdom SB, Stone L, Foster C, Galasko D, Llewellyn DM, Ramachandran VS: Rehabilitation of hemiparesis after stroke with a mirror. Lancet 1999, 353:2035-2036.

21. Ramachandran VS, Rogers-Ramachandran D: Synaesthesia in phantom limbs induced with mirrors. Proc Biol Sci 1996, 263:377-386.

22. Ramachandran VS, Rogers-Ramachandran D, Cobb S: Touching the phantom limb. Nature 1995, 377:489-490.

23. Oldfield RC: The assessment and analysis of handedness: the Edinburgh inventory. Neuropsychologia 1971, 9:97-113.

24. Kida T, Wasaka T, Inui K, Akatsuka K, Nakata H, Kakigi R: Centrifugal regulation of human cortical responses to a task-relevant somatosensory signal triggering voluntary movement. Neurolmage 2006, 32:1355-1364.

25. Hamalainen $\mathrm{M}$, Hari $\mathrm{R}$, IImoniemi R, Kunuutila J, Lounasmaa OV: Magnetoencephalography-Theory, instrumentation, and application to noninvasive studies of the working human brain. Rev Mod Phys 1993, 65:413-497.

26. Tipper SP, Lloyd D, Shorland B, Dancer C, Howard LA, McGlone F: Vision influences tactile perception without proprioceptive orienting. Neuroreport 1998, 9:1741-1744.

27. Kennett S, Taylor-Clarke M, Haggard P: Noninformative vision improves the spatial resolution of touch in humans. Curr Biol 2001, 11:1188-1191.

28. Longo MR, Pernigo S, Haggard P: Vision of the body modulates processing in primary somatosensory cortex. Neurosci Lett 2011, 489:159-163.

29. Kakigi R, Koyama S, Hoshiyama M, Watanabe S, Shimojo M, Kitamura Y: Gating of somatosensory evoked responses during active finger movements magnetoencephalographic studies. J Neurol Sci 1995, 128:195-204.

30. Kakigi R, Shimojo M, Hoshiyama M, Koyama S, Watanabe S, Naka D, Suzuki $H$, Nakamura A: Effects of movement and movement imagery on somatosensory evoked magnetic fields following posterior tibial nerve stimulation. Brain Res Cogn Brain Res 1997, 5:241-253.

31. Rushton DN, Rothwell JC, Craggs MD: Gating of somatosensory evoked potentials during different kinds of movement in man. Brain 1981, 104:465-491.

32. Wasaka $T$, Hoshiyama M, Nakata H, Nishihira Y, Kakigi R: Gating of somatosensory evoked magnetic fields during the preparatory period of self-initiated finger movement. Neurolmage 2003, 20:1830-1838.

33. Wasaka T, Kida T, Nakata H, Akatsuka K, Kakigi R: Characteristics of sensorimotor interaction in the primary and secondary somatosensory cortices 
in humans: a magnetoencephalography study. Neuroscience 2007, 149:446-456.

34. Wasaka T, Nakata H, Akatsuka K, Kida T, Inui K, Kakigi R: Differential modulation in human primary and secondary somatosensory cortices during the preparatory period of self-initiated finger movement. Eur $\int$ Neurosci 2005, 22:1239-1247.

35. Desmurget $\mathrm{M}$, Grafton $\mathrm{S}$ : Forward modeling allows feedback control for fast reaching movements. Trends Cogn Sci 2000, 4:423-431.

36. von Holst E, Mittelstaedt H: Das Reafferenzprinzip: Wechselwirkungen Zwischen Zentralnervensystem und Peripherie. Natursissenschaften 1950, 37:464-476.

37. Sperry RW: Neural basis of the spontaneous optokinetic response produced by visual inversion. J Comp Physiol Psychol 1950, 43:482-489.

38. Blakemore SJ, Goodbody SJ, Wolpert DM: Predicting the consequences of our own actions: the role of sensorimotor context estimation. J Neurosci 1998, 18:7511-7518.

39. Blakemore SJ, Wolpert DM, Frith CD: Central cancellation of self-produced tickle sensation. Nat Neurosci 1998, 1:635-640

40. Hesse MD, Nishitani N, Fink GR, Jousmaki V, Hari R: Attenuation of somatosensory responses to self-produced tactile stimulation. Cereb Cortex 2010, 20:425-432

41. Miall RC, Christensen LO, Cain O, Stanley J: Disruption of state estimation in the human lateral cerebellum. PLoS Biol 2007, 5:e316.

42. Fourneret $P$, Jeannerod $M$ : Limited conscious monitoring of motor performance in normal subjects. Neuropsychologia 1998, 36:1133-1140.

43. Farrer C, Frey SH, Van Horn JD, Tunik E, Turk D, Inati S, Grafton ST: The angular gyrus computes action awareness representations. Cereb Cortex 2008, 18:254-261.

44. Farrer C, Franck N, Georgieff N, Frith CD, Decety J, Jeannerod M: Modulating the experience of agency: a positron emission tomography study. Neurolmage 2003, 18:324-333.

45. Farrer $C$, Frith $C D$ : Experiencing oneself vs another person as being the cause of an action: the neural correlates of the experience of agency. Neurolmage 2002, 15:596-603.

46. Karnath HO, Baier B: Right insula for our sense of limb ownership and self-awareness of actions. Brain Struct Funct 2010, 214:411-417.

47. Karnath HO, Baier B, Nagele T: Awareness of the functioning of one's own limbs mediated by the insular cortex? J Neurosci 2005, 25:7134-7138.

48. Inui K, Wang X, Qiu Y, Nguyen BT, Ojima S, Tamura Y, Nakata H, Wasaka T, Tran TD, Kakigi R: Pain processing within the primary somatosensory cortex in humans. Eur J Neurosci 2003, 18:2859-2866.

doi:10.1186/1471-2202-13-138

Cite this article as: Wasaka and Kakigi: The effect of unpredicted visual feedback on activation in the secondary somatosensory cortex during movement execution. BMC Neuroscience 2012 13:138.

\section{Submit your next manuscript to BioMed Central and take full advantage of:}

- Convenient online submission

- Thorough peer review

- No space constraints or color figure charges

- Immediate publication on acceptance

- Inclusion in PubMed, CAS, Scopus and Google Scholar

- Research which is freely available for redistribution

Submit your manuscript at www.biomedcentral.com/submit
Biomed Central 